\title{
Hacia la erradicación de la poliomielitis: logros y retos en México
}

\author{
Alejandra Esteve-Jaramillo, MSP,(I) Vesta L Richardson López-Collada, MC.(I)
}

\section{Esteve-Jaramillo A, Richardson-López Collada VL. Hacia la erradicación de la poliomielitis: logros y retos en México. \\ Salud Publica Mex 20I2;54:537-543.}

\section{Resumen}

Desde la implementación de las estrategias globales de erradicación, la incidencia de parálisis poliomielítica ha disminuido dramáticamente. Cuatro estrategias han contribuido notablemente: a) Altas coberturas de inmunización con vacuna oral de polio (VOP), b) Inmunización suplementaria durante los Días Nacionales de Vacunación, c) Vigilancia epidemiológica efectiva de casos de parálisis flácida aguda (PFA), y d) Bloqueos vacunales en zonas de alto riesgo. Sólo quedan tres países polioendémicos, no obstante, cualquier país corre el riesgo potencial de importación del virus de algunas de estas áreas, de la liberación accidental del virus resguardado en laboratorios de diagnóstico clínico o investigación, o de la presencia de virus circulantes derivados de vacuna en el medio ambiente. Este documento pretende exponer los antecedentes históricos que hicieron posible la eliminación de la enfermedad en México, así como los retos para lograr un mundo libre de poliomielitis.

Palabras clave: poliomielitis; inmunización; eliminación; erradicación; México
Esteve-Jaramillo A, Richardson-López Collada VL.

Towards the eradication of poliomyelitis:

Mexico's achievements and challenges.

Salud Publica Mex 20I2;54:537-543.

\section{Abstract}

Since the strategies to eradicate polio were implemented, the incidence of paralytic polio has dropped dramatically. Four main strategies have greatly contributed: a) High immunization coverage rate with oral polio vaccine (OPV), b) Supplementary immunization activities during the $\mathrm{Na}$ tional Immunizations Days c) An effective epidemiological surveillance system for acute flaccid paralysis (AFP) and d) Intensified immunization activities in high risk areas. Three countries remain polio endemic, nevertheless, any country has a potential risk of the virus importation from one of these endemic areas; an accidental release of poliovirus from a research or clinical laboratory, or from having a circulating vaccine-derived poliovirus in the environment. The present document aims to provide an historical background that made possible the disease elimination in Mexico. Moreover, we discuss the challenges that every country needs to face in order to achieve a polio-free world.

Key words: poliomielitis; immunization; elimination; erradication; Mexico

(I) Centro Nacional para la Salud de la Infancia y la Adolescencia. Secretaría de Salud. México DF.

Fecha de recibido: 2 de enero de 2012 - Fecha de aceptado: 24 de mayo de 2012

Autor de correspondencia: Dra. Vesta L. Richardson López-Collada. Centro Nacional para la Salud de la Infancia y la Adolescencia. Secretaría de Salud. Francisco de P. Miranda 177, I ${ }^{\text {er }}$ piso, col. Merced Gómez. 01600 México DF, México.

Correo electrónico: vrichardson@salud.gob.mx 
$\mathrm{E}^{\mathrm{n}}$ 1985, el Club Rotario Internacional inició el programa Polio Plus, iniciativa del doctor Carlos Canseco González, médico mexicano presidente del club en esa época. El programa tenía el objetivo de inmunizar contra la polio a todos los niños del mundo para 2005. En este mismo año, la Organización Panamericana de la Salud (OPS) propuso la interrupción de la transmisión autóctona de poliovirus salvaje en el hemisferio occidental para $1990 .{ }^{1}$ Inspirados por esta iniciativa, en 1988, durante la $41^{\circ}$ Asamblea Mundial de Salud, los participantes se comprometieron a erradicar la poliomielitis en el año 2000. 2,3 Un año después se aprobó el Plan de Acción Mundial para la Erradicación de la Polio, encabezado por la OMS, Club Rotario Internacional, los Centros para el Control y Prevención de Enfermedades de los Estados Unidos (CDC, por sus siglas en inglés) y el Fondo de las Naciones Unidas para la Infancia (UNICEF).

El primer gran logro fue en 1994, cuando la región de América quedó certificada como libre de poliovirus, seguido por la eliminación en la región del Pacífico Oeste en 2000 y en Europa en 2002. ${ }^{4-6}$ Sin embargo, la certificación de esta última se vio amenazada en 2010 cuando se presentaron en la República de Tayikistán 458 casos de parálisis flácida con aislamiento de poliovirus salvaje tipo 1, el cual estuvo relacionado con el virus de Uttar Pradesh, en India. ${ }^{7,8}$

Desde febrero de 2012 sólo Pakistán, Afganistán y Nigeria son países endémicos de polio. Sin embargo, en algunos países la transmisión de poliovirus salvaje se ha reestablecido como en Chad y la República Democrática del Congo.

Para lograr la certificación global de erradicación se han establecido los siguientes criterios: a) ausencia de poliovirus salvaje aislado de casos de parálisis flácida aguda (PFA) en individuos sanos o muestras ambientales en todas las regiones de la Organización Mundial de la Salud al menos por tres años, en presencia de vigilancia epidemiológica de alta calidad, b) la contención de todas las reservas de poliovirus salvaje que se encuentran almacenadas en los laboratorios. ${ }^{9}$

El objetivo de este trabajo es exponer las estrategias que favorecieron el éxito de México en la eliminación de la poliomielitis, así como los retos a que se enfrentan los países hasta lograr un mundo libre de polio.

\section{Estrategias de eliminación de poliovirus en México}

\section{La inmunización}

La primera vacuna contra poliovirus que se utilizó en México fue la de poliovirus inactivada (VIP) de Salk en 1956, que se aplicó hasta 1961.
A partir de 1957, los estudios de inmunización con vacuna oral de poliovirus (VOP) en México del Dr. Ramos Álvarez, junto con la información de estudios en Singapur, Checoslovaquia y la URSS, lograron demostrar la inocuidad de la vacuna en individuos inmunizados y sus contactos. ${ }^{10-12}$ Fue en 1963 que se promulgó el decreto presidencial para administrar tres dosis de VOP al nacimiento, a $\operatorname{los} 4$ y a $\operatorname{los} 8$ meses. Una vez que se demostró que los niños de 6 a 8 semanas de edad mostraban una repuesta inmunológica adecuada a VOP, independientemente de la presencia de un nivel moderado de anticuerpos maternos, la vacunación se recomendó a los 2, 4 y 6 meses.

Con la creación del Programa Nacional de Inmunizaciones en 1973 se inició la aplicación masiva de la vacuna, donde se logró inmunizar a más de $70 \%$ de los niños menores de 5 años, con un subsecuente descenso de la tasa de morbilidad por polio. ${ }^{13}$ Las acciones permanentes fueron reforzadas en 1980 con las Semanas Nacionales de Vacunación contra Poliomielitis. Seis años después se iniciaron las acciones de erradicación de poliomielitis mediante la implementación de los Días Nacionales de Vacunación (DNV) con el fin de interrumpir la circulación del virus salvaje mediante la vacunación indiscriminada de los menores de 5 años. Gracias a estas acciones, entre 1986 y 1987, los poliovirus 1 y 2 (PV1 y 2) habían dejado de circular, mientras que en 1988 solamente se aisló el serotipo 3 en los estados de Sonora, Sinaloa y Jalisco.

A partir de 2007, el Consejo Nacional de Vacunación (CONAVA) recomendó incorporar al Esquema Nacional de Vacunación la vacunación secuencial de VIP/VOP. La primera se administra en el programa permanente junto con la vacuna contra difteria, tétanos, pertussis y Haemophilus influenza del tipo b (DPaT/VIP+Hib) a niños de 2, 4, 6 y 18 meses. La VOP se aplica dos veces al año (durante la $1^{\mathrm{a}}$ y $2^{\mathrm{a}}$ Semanas Nacionales de Salud) a los niños mayores de 6 meses y menores de 5 años que hayan recibido al menos dos dosis de VIP.

Si bien la última Encuesta Nacional de Cobertura de 2010 informó que 69.8\% de menores de 2 años han recibido VIP, con variaciones de cobertura entre los estados, con intervalos entre $56.8 \%$ en Guanajuato y 91.7\% en Tlaxcala, durante las SNS, más de $80 \%$ de los estados han logrado aplicar la VOP a más de $90 \%$ de la población blanco a vacunar ${ }^{14}$ (figura 1 ).

Se han realizado al menos tres seroencuestas en México para conocer los niveles de anticuerpos en la población, tomando en cuenta que la inmunidad puede adquirirse después de una infección por el virus salvaje, por vacunación o por paso transplacentario de anticuerpos de una madre a su bebé. ${ }^{15-17}$

En 1986 se llevó a cabo un estudio para conocer los niveles de seroconversión en niños menores de 4 años 


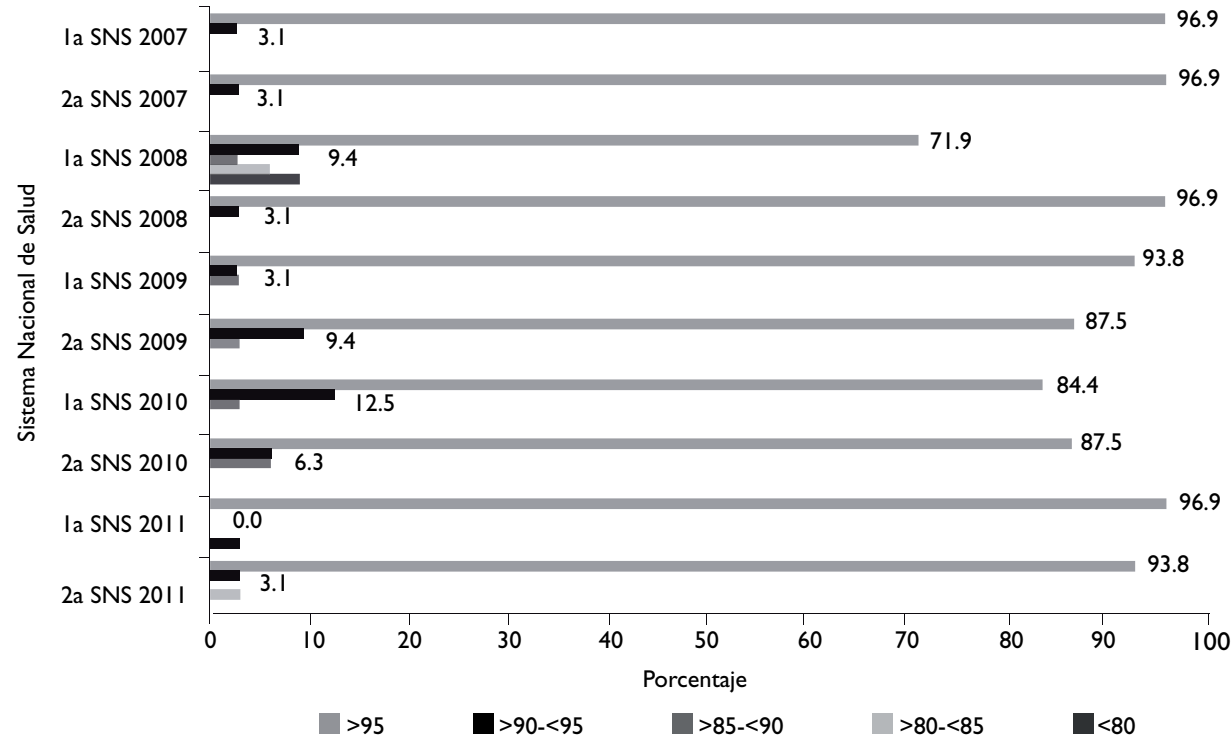

Fuente: Informe paralelo SENAS y fechas de ejecución Semanas Nacionales de Salud 2007-2010

Figura I. Distribución de los estados de la República mexicana, de acuerdo con el porcentaje de sus logros de vacunación con Vop, durante las Semanas Nacionales de Salud. Periodo 2007-2010

del estado de Chiapas. Se observó que previo a la vacunación, 77, 88 y 76\% eran seropositivos para PV1, PV2 y PV3, respectivamente. Este porcentaje se incrementó a más de $90 \%$ en el caso de PV1 y PV2, y a más de $80 \%$ para PV3, una vez que fueron inmunizados. ${ }^{15}$

En 1987 se realizó la Encuesta Nacional de Seroprevalencia, en donde se estudiaron sueros de 5260 niños entre 12 y 59 meses. ${ }^{16}$ Se encontraron anticuerpos antiPV1 en $92.7 \%$ de la población con al menos tres dosis de VOP, vs. $80.6 \%$ en los no inmunizados ( $p$ $=<.0001)$. La seroconversión para PV2 fue de $98.6 \mathrm{vs}$. 94.1\% $(p=<.0001)$, mientras que para PV3 fue 88.8 vs. $74.1 \%(p=<.0001)$.

En 2000 se efectuó una seroencuesta epidemiológica para conocer la prevalencia de anticuerpos contra poliovirus tipo 1 en la población menor de 10 años en México y se encontró una seropositividad de $99.3 \%$ (IC95\% 99.1-99.7). ${ }^{17}$

Posterior al cambio de la política de vacunación a un esquema secuencial de VIP/VOP, no se ha realizado una encuesta de seroprevalencia para conocer la población susceptible, principalmente en áreas que han reportado bajas coberturas de vacunación.

\section{Riesgos potenciales de la aplicación de la vacuna oral de poliovirus}

Si bien la VOP ha sido indispensable en la eliminación de polio debido a su bajo costo, a su alta inmunogenicidad, a su fácil aplicación y a su efecto de inmunidad de rebaño que favorece la inmunización de contactos no vacunados, desde que esta vacuna empezó a utilizarse ha habido informes esporádicos de casos de poliomielitis paralítica poco tiempo después de que el caso o sus contactos recibieron VOP, así como brotes de poliovirus derivados de vacunas circulantes. ${ }^{18-19}$ Por lo tanto, el uso sistemático de la VOP deberá abandonarse en todo el mundo después de que se haya detenido la transmisión del virus natural de poliomielitis y cuando la inmunidad de la población y la sensibilidad de la vigilancia epidemiológica sean altas. ${ }^{20}$

La poliomielitis paralítica asociada con vacuna (PPAV) se ha definido operacionalmente como una parálisis flácida aguda de una o más extremidades con disminución o ausencia de reflejos de estiramiento muscular (REMS) pero sin pérdida sensorial, en donde no haya otra causa aparente de la parálisis. Los casos de 
PPAV pueden clasificarse de acuerdo con el antecedente de haber recibido o no la vacuna. Un caso se considera "receptor" cuando la parálisis aparece 4 a 30 días posteriores de haber recibido VOP. Un caso es "contacto" cuando la parálisis ocurre 4 a 75 días después de que una persona con la que se haya tenido contacto en los 30 días previos a la parálisis haya recibido VOP. Puede presentarse un déficit neurológico 60 días después o defunción sin que haya otra causa aparente. Se requiere además el aislamiento de poliovirus vacunal en heces recolectadas hasta 14 días con posterioridad al inicio de la parálisis. ${ }^{21-23}$

Se calcula que el peso de esta enfermedad en el plano mundial es de 250 a 500 casos anuales, pero la incidencia informada ha sido variable de acuerdo con la metodología empleada para medirla. En México se informó de dos casos de PPAV en 1990 y 1994, y dos posteriores a la certificación en América: en 2004 en el estado de Tabasco y en 2006 en Hidalgo.

Otra complicación potencial es la aparición de poliovirus derivados de alguna de las tres cepas atenuadas incluidas en la vacuna oral, que presentan más de $1 \%$ de divergencia genética con la cepa prototipo del virus vacunal original en la secuencia de nucleótidos del gen de la proteína mayoritaria de la cápside (PV1). ${ }^{24}$ Los cambios acumulados en los nucleótidos de la proteína PV1 se producen con una tasa de mutación constante (1\% anual aproximadamente), hecho que permite estimar el tiempo que el virus lleva replicando y circulando. Estos cambios frecuentemente se asocian con la reversión de las cepas a fenotipos salvajes que recuperan su neurovirulencia, pueden presentar mayor capacidad de transmisión y de producción de enfermedad paralítica y han causado brotes de poliomielitis en poblaciones con bajas coberturas vacunales. Se presentaron 21 casos de poliovirus derivados de vacuna circulantes (cPVDV) tipo 1 en América: en República Dominicana y Haití durante 2000 y $2001 .{ }^{25}$

Uno de los objetivos de la erradicación es verificar la ausencia de poliovirus derivados de vacuna (PVDV) después de la certificación, cuando se ha suspendido el uso de VOP. Para conocer la emergencia de PVDV circulantes (cPVDV) se ha utilizado el monitoreo ambiental de aguas residuales. En países que han cambiado sus políticas de inmunización de VOP por VIP, o esquemas secuenciales VIP/VOP, este proceso tiene el fin de identificar si la duración de la excreción del virus Sabin es menor en niños previamente inmunizados con VIP, y la capacidad del virus de convertirse en un cPVDV. ${ }^{26} \mathrm{En}$ México no se cuenta en la actualidad con un sistema de vigilancia ambiental de poliovirus, pero se han realizado estudios de investigación utilizando este método. Un ejemplo es un estudio transversal realizado en Orizaba,
Veracruz, en donde se recolectaron muestras de heces de niños y de aguas residuales de 6 a 13 semanas posteriores a una de las SNS en donde se aplicó Sabin. ${ }^{27}$ Utilizando la técnica de reacción en cadena de la polimerasa en tiempo real (RT-PCR), se demostró la circulación de VOP incluso 13 semanas después de la vacunación. Más aún, se detectó una proporción revertante para polio Sabin tipo $1,>0.5$ en ambos tipos de muestras recolectadas (una proporción revertante de 1.0 es indicativo de que la mayoría de los virus tiene mutaciones puntuales en la región no traducida 5' que se asocia a PPAV), mientras que Sabin tipo 2 y 3 fueron no revertantes.

\section{Riesgos de la suspensión de la VOP}

El cese de la vacunación con VOP tampoco está exento de riesgos, ya que modelos matemáticos han sugerido que con el cese simultáneo de administración en el ámbito mundial hay un riesgo de $60-95 \%$ de al menos un brote de CPVDV en algún lugar del mundo durante los primeros 12 meses inmediatamente después del cese de VOP. Este riesgo se reducirá a 1-6\% después de 36 meses. ${ }^{28} \mathrm{El}$ tema ha reunido a expertos mundiales para discutir sobre el tiempo y estrategias necesarias para detener la producción y el uso de VOP en el mundo de manera coordinada, una vez que se detecte el último caso de poliomielitis por virus salvaje y se haya logrado la certificación de un mundo libre de polio. 20,29,30

Actualmente se trabaja en nuevos instrumentos de vacunación como preparaciones monovalentes de Sabin y una vacuna inactivada producida con cepas atenuadas derivadas de VOP. ${ }^{20,31}$

\section{El sistema de vigilancia de parálisis flácida aguda}

Mantener adecuada vigilancia de la parálisis flácida aguda es indispensable para poder establecer una respuesta eficaz a cualquier brote resultante de la importación de poliovirus salvaje, cPVDV o liberación accidental de poliovirus a partir de un laboratorio o unidad de investigación.

En México, el Sistema Nacional de Vigilancia Epidemiológica incluyó a la poliomielitis para su notificación inmediata y obligatoria a partir de 1965. Bajo el sistema de vigilancia activa para casos de parálisis flácida aguda, se logró identificar el último caso de poliomielitis salvaje en Tomatlán, Jalisco, el 18 de octubre de 1990. En este caso se aisló el poliovirus tipo 3 .

Para evaluar la sensibilidad de los sistemas de vigilancia epidemiológica se han establecido algunos criterios. Estos son: a) la detección de al menos un caso anual de PFA por cada 100000 habitantes menores de 15 años, b) que al menos $80 \%$ de las muestras colectadas de 
los casos de PFA sean adecuadas, c) que estas muestras sean analizadas en un laboratorio acreditado por la OMS (figura 2).

\section{Red de laboratorios de diagnóstico}

En 1989 se creó un sistema de red global de laboratorios que apoyaría la iniciativa de erradicación de polio. Desde entonces la participación del laboratorio en el proceso de erradicación ha sido crucial. Esta red está conformada por tres niveles con responsabilidades específicas para cada uno: a) laboratorios mundiales especializados, b) laboratorios de referencia regional, c) laboratorios nacionales. Todos ellos trabajan coordinadamente en la red de laboratorios de salud pública más grande que jamás se haya visto. ${ }^{33}$

Para mantener la vigilancia, en casos de poliomielitis debe analizarse una muestra suficiente de heces (entre 8 y 10 gramos) de pacientes con PFA dentro de los primeros 14 días posteriores al inicio de la parálisis. Las muestras deben transportarse en un plazo de 72 horas posteriores a su obtención y es esencial mantener una adecuada red fría (entre 4 y $8^{\circ} \mathrm{C}$ ). El aislamiento primario del virus se realiza en el Laboratorio de Poliovirus del Instituto de Diagnóstico y Referencia Epidemiológica (InDRE) en la Ciudad de México. Si se obtiene un aislamiento positivo para poliovirus, la muestra se envía para su diferenciación intratípica al Laboratorio de Pruebas Molecular del InDRE. De esta forma se caracterizará si este poliovirus es salvaje (non-Sabin like) o de cepa vacunal (Sabin-like). En caso de estos últimos, se utilizan primers específicos para diferenciar también a los PVDV, que se envían para secuenciación genética a los CDC.

La contención y resguardo del poliovirus en el laboratorio

La contención de materiales infecciosos de poliovirus salvaje es esencial después de la erradicación para reducir al mínimo el riesgo de liberación accidental y reintroducción de poliovirus salvaje desde los laboratorios hacia la comunidad.

En 2004 se creó la Comisión Regional de América (CRCAM) para la contención del virus salvaje de la poliomielitis y verificar que América se mantiene libre

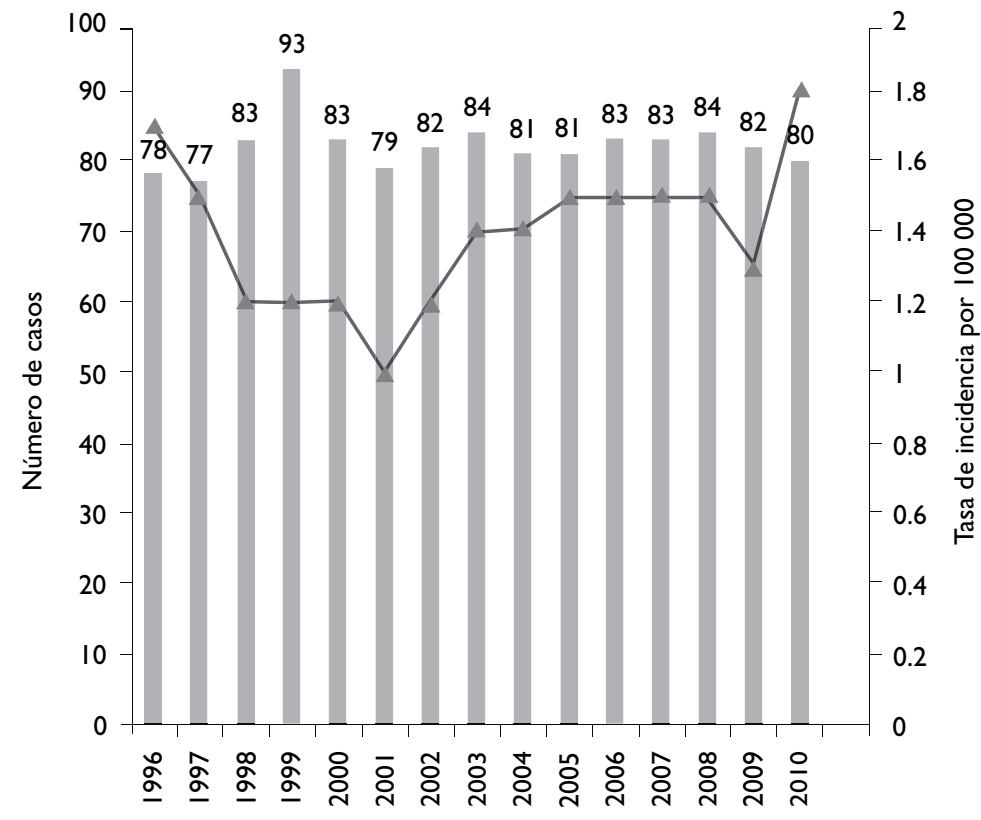

- Casos de PFA con muestra adecuada*

- Tasa de PFA no causada por polio

$*$ Objetivo $\geq 80 \%$

‡ Objetivo: $\geq 1 / 100,000$

Referencia 32

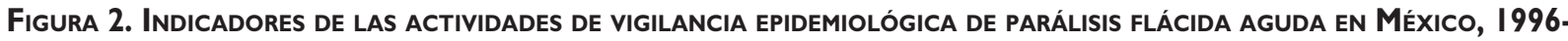
2010 
de poliomielitis. Ésta vigila el cumplimiento de las acciones establecidas en el plan de contención del virus de la polio en los laboratorios de la GCC.

Este plan contempla tres fases: a) Encuesta e inventario de laboratorios que resguardan poliovirus salvaje y establecimiento de un plan para destruir o transportar estos materiales a un laboratorio de alto nivel de bioseguridad, b) Certificación global, c) Postcertificación global. ${ }^{34}$

En abril de 2010, América recibió la certificación de haber concluido exitosamente la fase 1, en donde cada país realizó un inventario de laboratorios que contienen virus salvajes de poliomielitis. En los 43 países de la región se identificaron 67362 laboratorios e instituciones y se encuestó a 59618 (88.5\%). El virus salvaje de la polio o la existencia de material potencialmente infeccioso se encontró en 215 laboratorios e instituciones en nueve países. En México se notificaron cuatro de estas instituciones.

\section{Discusión}

El proceso de erradicación ha sido muy dinámico y ha propuesto nuevos retos. Pero gracias a la cooperación de organizaciones y gobiernos en el ámbito mundial se han superado ya muchos de ellos.

Aún quedan casos de poliovirus en el mundo, lo cual representa una amenaza potencial para cualquier país por el riesgo de liberación o importación del virus salvaje, así como de la posible aparición de un poliovirus derivado de la vacuna Sabin.

Debido a la importancia de lograr un mundo libre de polio y evitar la parálisis en millones de niños, es necesario mantener una vigilancia epidemiológica de calidad, incluyendo el monitoreo ambiental para la detección de PVDV. Asimismo se deben mantener niveles de inmunidad alta en la población, alcanzando coberturas de vacunación con VIP $\geq 95 \%$ en menores de 5 años (especialmente en menores de 1 año), así como coberturas de dosis adicionales de $\mathrm{VOP} \geq 95 \%$ en niños de 7 a 59 meses de edad.

Una vez que se haya detenido la transmisión del virus en todo el mundo, será necesario ajustar las políticas de vacunación que respondan a los objetivos de la fase poserradicación.

\section{Conclusiones}

Las estrategias de eliminación del poliovirus en México han sido exitosas, sin embargo es esencial mantener una vigilancia epidemiológica de calidad así como coberturas vacunales elevadas. Si bien se cuenta con estos dos indicadores en el ámbito nacional, es importante vigilar el desempeño a nivel estatal y jurisdiccional, ya que de no cumplirse existiría un riesgo de circulación de poliovirus salvaje o PVDV. Un método sensible que permitiría la identificación oportuna de estos agentes en las poblaciones, principalmente en aquellas con bajas coberturas de vacunación y servicios de salud escasos o deficientes, es la vigilancia de muestras ambientales. Finalmente, es esencial la integración y colaboración de todos los sistemas nacionales de vigilancia epidemiológica, inmunización y red de laboratorios para integrar la información que permita tomar decisiones oportunas encaminadas a la prevención de nuevos casos de poliomielitis en México.

Declaración de conflicto de intereses. Los autores declararon no tener conflicto de intereses.

\section{Referencias}

I. Pan American Health Organization. Director announces campaign to eradicate poliomyelitis from the Americas by 1990. Bull Pan Am Health Organ 1985;19:213

2. World Health Assembly. Global eradication of poliomyelitis by the year 2000. Geneva: World Health Organization, 1988; resolution WHA4I.28. 3. Robertson SE, Chan C, Kim-Farley R, Ward N. Worldwide status of poliomyelitis in 1986, 1987 and 1988, and plans for its global eradication by the year 2000. World Health Stat Q 1990;43:80-90.

4. Centers for Disease Control and Prevention (CDC). Certification of poliomyelitis eradication--the Americas, 1994. MMWR Morb Mortal Wkly Rep 1994; 43:720-722.

5. Centers for Disease Control and Prevention (CDC). Certification of poliomyelitis eradication--Western Pacific Region, October 2000. MMWR Morb Mortal Wkly Rep 200I; 50:I-3.

6. Centers for Disease Control and Prevention (CDC). Certification of poliomyelitis eradication--European Region, June 2002. MMWR Morb Mortal Wkly Rep 2002;5I:572-574.

7. World Health Organization. Country Office Tajikistan; WHO Regional Office for Europe; European Centre for Disease Prevention and Control. Outbreak of poliomyelitis in Tajikistan in 2010: risk for importation and impact on polio surveillance in Europe? Euro Surveill 2010;15.

8. Centers for Disease Control and Prevention (CDC). Outbreaks following wild poliovirus importations. Europe, Africa, and Asia, January 2009. September 2010. MMWR Morb Mortal Wkly Rep 2010; 59:1393-1399.

9. Smith J, Leke R, Adams A, Tangermann RH. Certification of polio eradication: process and lessons learned. Bull World Health Organ 2004; 82:24-30.

II. Sabin AB. Properties and behavior of orally administered attenuated poliovirus vaccine. J Am Med Assoc 1957; 164: I216-1223.

10. Sabin AB. Status of field trials with an orally administered, live attenuated poliovirus vaccine. J Am Med Assoc 1959; 171:863-868.

12. Ramos-Alvarez M, Bustamante ME, Alvarez Alba R. The use of the Salk vaccine, in the polio live virus, in Mexico: Study Results. Bol Oficina Sanit Panam 1961; 50:305-322.

I3. Consejo Nacional de Vacunación. Manual de vacunación 2008-2009. México, D.F: CONAVA 2008: 405.

14. Instituto Nacional de Salud Pública. Encuesta Nacional de Cobertura de Vacunación. Cuernavaca, Morelos: INSP, 2010: 54.

15. Díaz-Ortega J, Valdespino JL, Zárate ML, Camacho L. Seroconversión a la vacuna Sabin trivalente en niños menores de 4 años de edad. Bol Hosp Inf Mex 1989; 46:517-520. 
16. Ruiz-Gómez J, Tapia-Conyer R, Salvatierra B, et al. Seroepidemiología de la poliomielitis en México. Salud Publica Mex 1992; 34:168-176.

17. Ruiz-Gómez J, Valdespino JL, Olaiz-Fernández G, Arias-Toledo E, Sepúlveda J. Seroprevalencia de anticuerpos contra el poliovirus I en niños mexicanos. Salud Publica Mex 2007;49:S36I-S369.

18. Dowdle WR, De Gourville E, Kew OM, Pallansch MA, Wood DJ. Polio eradication: the OPV paradox. Rev Med Virol 2003;13(5):277-29I.

19. Aylward RB, Sutter RW, Heymann DL. Policy: OPV cessation - the final step to a 'polio-free' world. Science 2005; 310: 625-626.

20. Global Polio Eradication Initiative. Framework for National Policy Makers in OPV-Using Countries [internet]. Ginebra, (Suiza): World Health Organization; 2008 Dec [consultado 30 de noviembre de 20II]

I lp. Disponible en: http://www.polioeradication.org/content/publications/ OPVCessationFrameworkEnglish.pdf

21. Albrecht RM. Poliomyelitis from a vaccinee. Lancet 1968; I:I37I. 22. Nkowane BM, Wassilak SGF, Orenstein WA, et al. Vaccine associated paralytic poliomyelitis, United States: 1961-72. JAMA 1987; 257: 1335-1340. 23. Esteves K. Safety of oral poliomyelitis vaccine: results of a WHO enquiry. Bull World Health Organ 1988; 66:739-746.

24. Kew OM, Wright PF, Agol VI, Delpeyroux F, Shimizou H, Nathanson $\mathrm{N}$ et al. Circulating vaccine-derivated polioviruses: current state of knowledge. Bull World Health Organ 2004; 82:16-23.

25. CDC. Outbreak of poliomyelitis - Dominican Republic and Haiti, 2000. MMWR Morb Mortal Wkly Rep 2000;49: 1094-I 103.

26. Zurbriggen S, Tobler K, Abril C, Diedrich S, Ackermann M, Pallansch $\mathrm{MA}$, et al. Isolation of sabin-like polioviruses from wastewater in a country using inactivated polio vaccine. Appl Environ Microbiol 2008; 74:5608-56|4.
27. Troy SB, Ferreyra-Reyes L, Huang C, Mahmud N, Lee YJ, CanizalesQuintero $S$. Use of a novel real-time PCR assay to detect oral polio vaccine shedding and reversion in stool and sewage samples after a mexican national immunization day. J Clin Microbiol 20 I I;49: I777-I783.

28. Duintjer-Tebbens RJ, Pallansch MA, Kew OM, Cáceres VM, Sutter RW, Thompson KM. A dynamic model of poliomyelitis outbreaks: learning from the past to help inform the future. Am J Epidemiol 2005; 162:358-372.

29. Salas-Peraza D, Avila-Agüero ML, Morice-Trejos A. Switching from OPV to IPV: are we behind the schedule in Latin America? Expert Rev Vaccines 2010;9:475-483.

30. Sutter R, Prevots DR, Cochi SL. Introduction of inactivated poliovirus vaccine into oral polio vaccine-using countries. Wkly Epidemiol. Rec 2003; 78: 24I-252.

31. Verdijk P, Rots NY, Bakker WA. Clinical development of a novel inactivated poliomyelitis vaccine based on attenuated Sabin poliovirus strains. Expert Rev Vaccines 201 I; 10:635-644.

32. World Health Organization. Global Polio Erradication Initiative [Internet] Ginebra (Suiza) 1996- [Consultado 30 de marzo 2012]. Disponible en: http://apps.who.int/immunization_monitoring/en/diseases/poliomyelitis/ case_count.cfm

33. Arya SC, Agarwal N. Global polio laboratory network: future pursuit and commitments. J Clin Virol 2007;38:362-363.

34. Completion of national laboratory inventories for wild poliovirus containment:WHO Region of the Americas, March 2010. Wkly Epidemiol Rec 2010; 85:329-333. 